\title{
Pilot study of a strap-based custom wheelchair seating system in persons with spinal cord injury
}

\author{
John E. Ferguson, PhD; ${ }^{1-2 *}$ Becky L. Wittig, PT, ATP; ${ }^{1}$ Mark Payette, CO, ATP; ${ }^{3}$ Gary D. Goldish, MD; ${ }^{1-2}$ \\ Andrew $\mathbf{H}$. Hansen, $\mathbf{P h D}^{\mathbf{1 - 2}}$ \\ ${ }^{1}$ Minneapolis Department of Veterans Affairs Health Care System, Minneapolis, MN; ${ }^{2}$ Department of Physical Medicine and \\ Rehabilitation, University of Minnesota, Minneapolis, MN; ${ }^{3}$ Tamarack Habilitation Technologies Inc, Minneapolis, MN
}

\begin{abstract}
Custom wheelchair seats can be used to help prevent pressure ulcers in individuals with spinal cord injury. In this study, a strap-based system was evaluated in three Veterans with spinal cord injury. Interface pressure distributions were measured after transfers, wheeling, and pressure relief maneuvers and after fittings by three different therapists. We found that pressure distribution measures were not generally affected after transfers and wheeling using the strap-based wheelchair and that pressure relief maneuvers were able to be performed. Additionally, all therapists were able to customize the wheelchair seat to clinically acceptable levels in 4 to $40 \mathrm{~min}$ for the three subjects. Future studies can test the long-term effects of using the strap-based wheelchair seat and identify individuals that would most benefit from a rapidly customizable wheelchair seat.
\end{abstract}

Key words: custom seating, custom wheelchair, interface pressure mapping, pressure relief maneuvers, pressure ulcer, SCI, spinal cord injuries, transfers, ulcer prevention, wheelchairs.

\section{INTRODUCTION}

Pressure ulcers are a common and serious complication of spinal cord injury (SCI) [1-3]. Because individuals with SCI frequently spend several hours daily seated in wheelchairs, pressure ulcers often develop over bony prominences that sustain large loads during sitting, such as the ischial tuberosities and the coccyx. Using a wheelchair seat that redistributes interface pressures is an important component of a pressure-ulcer prevention strategy [4].
The process of selecting a seating surface for a wheelchair requires considering many factors. These factors include the distribution of pressure and shear stresses on soft tissues, moisture accumulation, heat accumulation or heat loss, stability, thickness, durability, cost, and appearance [5]. Each individual's needs are different, and there is no single wheelchair cushion that works for all wheelchair users [6].

While selecting or adjusting a wheelchair cushion, therapists often use interface pressure mapping (IPM) to visualize the distribution of interface pressures in real time. IPM can be used to calculate several measurement variables to quantify the quality of pressure distribution of the seating surface. Sprigle et al. found three such measures to be clinically useful and reliable: peak pressure index (PPI), dispersion index (DI), and contact area (CA) [7]. PPI is the highest average pressure across a load-bearing area, such as under an ischial tuberosity. DI is the percentage of the total pressure that is supported by

\footnotetext{
Abbreviations: $\mathrm{CA}=$ contact area, $\mathrm{DI}=$ dispersion index, $\mathrm{IPM}=$ interface pressure mapping, $\mathrm{PPI}$ = peak pressure index, $\mathrm{SCI}=$ spinal cord injury, $\mathrm{T}=$ thoracic, $\mathrm{VA}=$ Department of Veterans Affairs.

*Address all correspondence to John E. Ferguson, PhD; Minneapolis VA Health Care System, One Veterans Drive (151), Minneapolis, MN 55417; 612-629-7471; fax 612-7252093. Email: john.ferguson1@va.gov http://dx.doi.org/10.1682/JRRD.2014.01.0007
} 
the sacrococcygeal and ischial zones. CA is the total area of the pressure sensors that are under load.

Using IPM properly requires a few important considerations. IPM is a valuable clinical tool to evaluate seating options for a single person, but it should not be used to compare across individuals. Although lower PPI, lower DI, and higher CA values are generally preferred, there are no ideal values. Measurements of PPI, DI, or CA that are within repeatability coefficients of $17.7 \mathrm{~mm}$ $\mathrm{Hg}, 7$ percent, or $23.7 \mathrm{in}^{2}$, respectively, of each other can be considered to be equivalent [7]. It is also important to note that pressure distribution measurements can be significantly affected by the type of cushion used and the pressure sensor itself [8]. Additionally, an improvement in one measurement variable at the expense of the others does not necessarily represent a better pressure distribution. Ultimately, clinical judgment is the most important deciding factor in evaluating a seating surface for a wheelchair.

If standard wheelchair cushions are deemed inadequate, individuals with SCI may require custom seating. Custom seats have individualized contoured seating surfaces, which help increase the contact area of seating and redistribute pressures away from the bony prominences [9-11]. This pressure redistribution would generally correspond to a decrease in PPI and DI and an increase in CA. Custom cushions can be made using many different materials and fabrication methods, including vacuumforming sheet plastics, vacuum-consolidated glass or Styrofoam beads, and molding gel or foam [5,12-13]. For many of these custom seats, the time required for customization is a large concern. Therapists may have to wait hours, days, or weeks before the custom cushion is ready. Faster custom seats, such as "foam in place" molding systems, can be ready in less than an hour but are complicated to use, have concerns over toxicity, and do not allow for readjustments to the seating contour [14].

The present study is the first clinical evaluation of a new, strap-based seating system (FlexForm seating system, Tamarack Habilitation Technologies Inc; Blaine, Minnesota) (Figure 1). The seating system is different in design and approach from existing custom-contoured seating surfaces. The lengths of the interwoven straps that make up the seating surface can be individually adjusted to create a custom seat contour. This study aimed to test the interface pressure distributions after subjects performed several common wheelchair activities, such as transfers, wheeling, and pressure relief maneuvers, and the interface pressure distributions after fittings by different therapists. The time required for therapists to perform fittings was also assessed. Overall, this pilot study aimed to evaluate the strap-based seating system's potential for clinical usage and to inform future research studies.

\section{METHODS}

\section{Strap-Based Wheelchair Seating System}

The FlexForm seating system is a prototype wheelchair with a strap-based seat (Figure 1(a)) that can be configured by a clinician using molding tools and a jack stand (Figure 1(b)). The seat has 13 horizontal straps and 13 longitudinal straps that are interwoven and connected directly to a custom-manufactured, aluminum wheelchair frame. On top of the straps is a seat cover made of a polyester spacer fabric layer and a nylon/spandex fabric layer with low-friction zones below the pelvis and greater trochanters. (a)

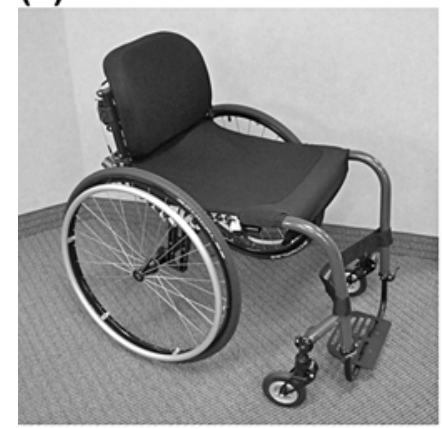

(c)

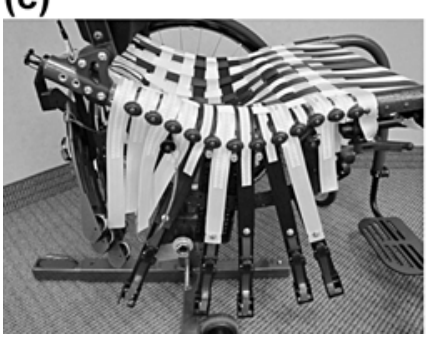

(b)

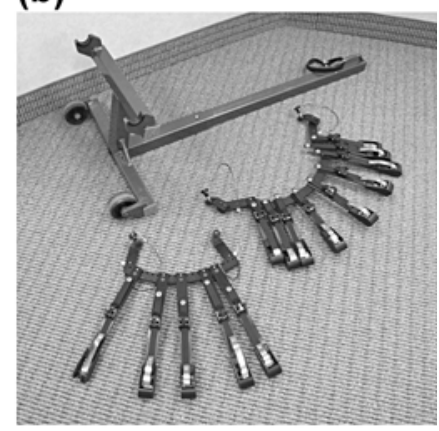

(d)

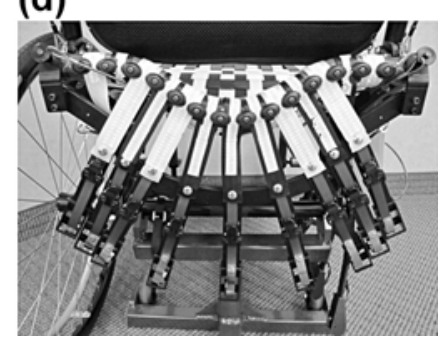

Figure 1.

FlexForm seating system. (a) Wheelchair with strap-based seat and cover. (b) Two molding tools, seen in foreground, which assist in strap length adjustments; jack stand, seen in background, which lifts wheelchair's wheels off ground to prevent wheelchair from moving during fitting process. Close-up view of molding tools temporarily connected (c) to horizontal straps and (d) to longitudinal straps during fitting process. 
The wheelchair seat is designed to be used without any additional cushions.

The straps can be individually lengthened or shortened, creating the custom-contoured support surface. To assist in adjusting the strap lengths, a jack stand is placed below the wheelchair frame and locked into place. Molding tools are then attached to the right (Figure 1(c)) and the back (Figure 1(d)) of the wheelchair. These molding tools use ratchet straps, buckles, and coil springs to change the strap lengths of five horizontal and nine longitudinal straps. The other strap lengths can be set manually. All the straps are locked into place using low-profile clamps. At the end of the fitting process, the molding tools and jack stand are removed.

\section{Subjects with Spinal Cord Injury}

Three Veteran subjects with SCI were recruited for the study. The subjects were manual wheelchair users and did not have existing pressure ulcers. All three subjects had complete SCIs (American Spinal Injury Association Impairment Scale A). Subject 1 was a 53 yr old male, $158 \mathrm{lb}$, 70.5 in. tall, and injured at the thoracic (T)5 level $31 \mathrm{yr}$ before the study. Subject 2 was a 54 yr old male, $179 \mathrm{lb}$, 66 in. tall, and injured at the T2 level 24 yr before the study. Subject 3 was a 64 yr old male, $207 \mathrm{lb}, 70$ in. tall, and injured at the T12 level $12 \mathrm{yr}$ before the study. Additionally, the subjects did not have any experience with the FlexForm seating system prior to this study. All Veteran subjects completed the consent process approved by the Institutional Review Board of the Minneapolis Department of Veterans Affairs (VA) Health Care System.

\section{Therapist Subjects}

Five therapist subjects, including both physical therapists and occupational therapists, were also recruited to participate in the study. The therapists worked at the Minneapolis VA Health Care system, and each had over a year experience fitting wheelchair seats for patients with SCIs. Before the study, the therapists were trained on how to use the FlexForm seating system by the manufacturer during an approximately $2 \mathrm{~h}$ session. All therapist subjects completed the consent process approved by the Institutional Review Board of the Minneapolis VA Health Care System.

\section{Fitting Process}

For the purposes of the study, the fitting process was divided into two parts: an initial fitting and a final fitting. The initial fitting process created a quick custom contour without adjusting the straps individually. The final fitting process allowed for fine-tuning of the straps by the therapist.

To perform the initial fitting process, therapists first assisted the subject in transferring to the FlexForm wheelchair. Then, the therapist reset the straps to their default lengths while the subject performed a wheelchair push-up or a forward-lean. Then, the therapist asked the subject to carefully lower himself onto the seat, which resulted in the straps lengthening different amounts based on the load distribution and on different tension coil springs. Next, the subject was asked to reach to the right and to the left and lean forward and backward. The subject's upper legs were also lifted toward his chest, flexing the hips. The subject then wheeled across the room and back a few times, turning sharply to the left and to the right. By this time, the straps were not lengthening any more and an initial contour of the seating surface was formed. The time required to perform the initial fitting process was noted, beginning when the subject lowered himself onto the chair and ending when the therapist decided the straps were not lengthening any more. A pressure mat was then placed under the subject to assess the pressure distribution.

To perform the final fitting process, therapists first set the wheelchair on a jack stand to prevent it from rolling during fine-tuning adjustments. The right wheel was removed to allow access to the side molding tools. For the straps that were connected to the molding tools, the therapist used the buckles to lengthen or to shorten the straps approximately $1 \mathrm{~mm}$ at a time. For the other straps, the therapist would unscrew the clamp holding the strap in place, lengthen or shorten the strap directly, and then screw the clamp back onto the strap. The therapist continued to adjust the individual strap lengths until the desired pressure distribution was reached. The time required to perform the final fitting process was noted, beginning when the therapist set the wheelchair on the jack stand and ending when the therapist finished the strap adjustments. At the end of the fitting process, the straps were locked in place with low-profile clamps, the molding tools and jack stand were removed, and the excess straps were tucked beneath the seating surface.

\section{Interface Pressure Mapping}

Pressure measurements were performed using the XSENSOR X3 LX100 flexible sensor mat (XSENSOR Technology Corporation; Calgary, Canada). The mat measured pressures at 1,296 points across an array of $36 \times 36$ capacitive pressure sensors, which covered an $18 \times 18$ in. sensing area. Subjects sat on the sensor mat during most 
of the study's activities (including the final fitting process, wheeling laps, transfers, and pressure relief maneuvers); the sensor mat was not used during the initial fitting process, and it was removed during transfers if the subject found it made transferring more difficult. Subjects were occasionally asked to perform wheelchair push-ups while the therapists repositioned the sensor mat to reduce folds as much as possible. Because of the thin, strap-based seat design, bony prominences could be palpated by a therapist from below the seat without affecting the subject's seated posture. Before each interface pressure measurement, a therapist would press into the FlexForm seat at the subject's left ischial tuberosity, right ischial tuberosity, coccyx, and the midline between the legs. These presses were recorded by the sensor mat and were used to define anatomical regions in the postprocessing of the interface pressure data. Because the wheelchair seat did not have a cushion, cushion-settling delays were not a concern. Pressure measurements were recorded once the subject and therapist were ready and in position.

\section{Study Procedure}

Testing for each subject took place during a single day, including a $3 \mathrm{~h}$ morning session and a $3 \mathrm{~h}$ afternoon session (Figure 2). All testing took place at the Minneapolis VA Health Care System using protocols approved by the local institutional review board.

In the morning session, testing focused on common wheelchair activities, including transfers, wheeling, and pressure relief maneuvers. The wheelchair's seat depth and footrest height were adjusted to fit the subject. Then, the seat was fit to the subject by both an experienced VA therapist (author B.W.) and an orthotist and coinventor of the FlexForm seat (author M.P.). The time required to complete the initial fitting process and the final fitting process were

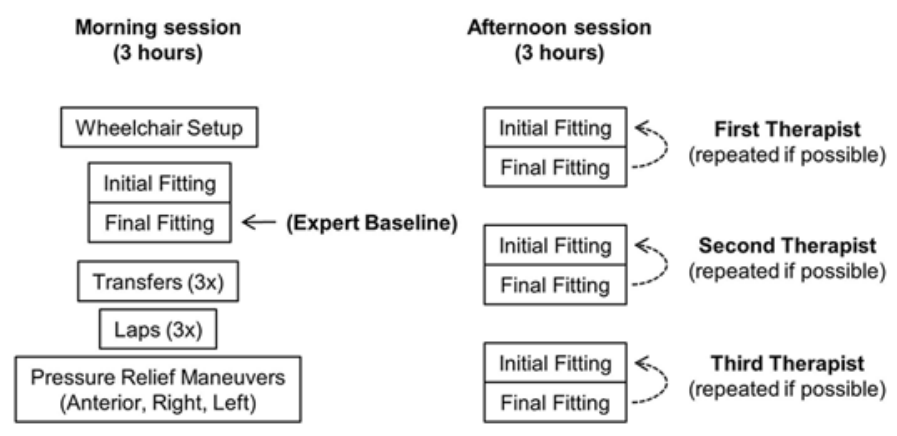

Figure 2.

Study protocol for three subjects with spinal cord injury. noted, and the seat was locked into place for the remainder of the morning session. At this time, interface pressures of the FlexForm wheelchair seat were recorded and would serve as an "expert baseline" for comparisons with other interface pressures measured for the subject. Next, the subject transferred three times from the wheelchair to a mat platform and back to the wheelchair. The pressure mat was removed from the wheelchair seat if the subject felt it made transfers more difficult. Interface pressures were recorded after each transfer and compared with the expert baseline. The subject then wheeled three laps at a comfortable, selfselected speed around a $280 \mathrm{ft}$ predefined route in the hospital hallways, including both left and right turns. After each lap, interface pressures were recorded and compared with the expert baseline. The subject then performed three pressure relief maneuvers once: an anterior pressure relief (by placing elbows on knees), a right pressure relief (by reaching to the right to grasp a handle by the floor), and a left pressure relief (by reaching to the left to grasp a handle by the floor). Interface pressures were recorded immediately before and after each pressure relief maneuver and compared with the expert baseline.

In the afternoon session, testing focused on fittings by three therapist subjects (chosen from therapists 1-5 based on availability). Each therapist fit the seating system for the subject as many times as possible in an hour. The straps were reset before each fitting. Therapists were instructed to modify the seat until they felt comfortable sending the subject home with the chair. Pressure mapping was used to assist with the fitting process. Interface pressures were measured after each initial and final fitting and compared with the expert baseline. The times to complete the initial and final fitting processes were also noted. All subjects went through the same order of procedures in the morning and afternoon session (except therapist 1 performed initial and final fittings for subject 3 in the morning after wheelchair setup, rather than the afternoon, because of scheduling issues).

\section{Data Analysis}

Three outcome variables were calculated from the interface pressure measurements, based on Sprigle et al. [7], using MATLAB (MathWorks; Natick, Massachusetts) and X3 Medical software (XSENSOR Technology Corporation). PPI was determined by finding the largest pressure measurement and taking the mean pressure of that point and the eight sensor points surrounding it. DI was calculated by finding the total pressure measured in a $7 \times$ 7 in. area centered on the ischial tuberosities and coccyx 
and dividing this by the total pressure measured across the whole mat. The $7 \times 7$ in. area was determined using the data from the therapist's pressure mat presses that were made before every interface pressure measurement. CA was calculated by finding the total sensor area that measured pressures of at least $5 \mathrm{~mm} \mathrm{Hg}$. These IPM outcome variables were used to compare the expert baseline with the pressure distribution measurements after wheeling laps and transfers and after initial and final fittings by the three therapist subjects. Pressure distributions were generally considered better or equivalent to the expert baseline if they had lower or equivalent PPIs, lower or equivalent DIs, and higher or equivalent CAs. Equivalent pressure distributions were within the reliability coefficient from each other (17.7 mm Hg, 7\%, and 23.7 in. $^{2}$ for PPI, DI, and CA, respectively). The IPM outcome variables were also used to assess the ability of subjects to perform pressure relief maneuvers in the wheelchair.

Also, the time required to complete the initial fitting process and the final fitting process were noted for all subjects. The total fitting time, which was the sum of the initial and final fitting times, was also noted. These times were compared across subjects to determine the average and range of times required to fit the wheelchair seat.

\section{RESULTS}

The three subjects with SCI were able to complete all portions of the study. All therapist subjects in the study were able to customize the wheelchair seat for the three subjects with SCI so that the pressure distributions were deemed acceptable based on clinical judgment.

\section{Transfers, Laps, and Pressure Reliefs}

All three subjects with SCI had consistent pressure distributions after transfers and laps around the hospital hallways. Figure 3 shows the pressure distribution measurements after each of three transfers to a mat platform and back to the wheelchair. Figure 4 shows the pressure distribution measurements after three laps around the hospital hallways. Almost all pressure distribution measurements were equivalent to or better than the expert baseline. The only exceptions were after subject 3's third transfer and third lap, which had higher PPIs than the expert baseline (18 and $32 \mathrm{~mm} \mathrm{Hg}$ higher, respectively).

All three subjects with SCI were able to successfully perform pressure relief maneuvers on the FlexForm wheel-

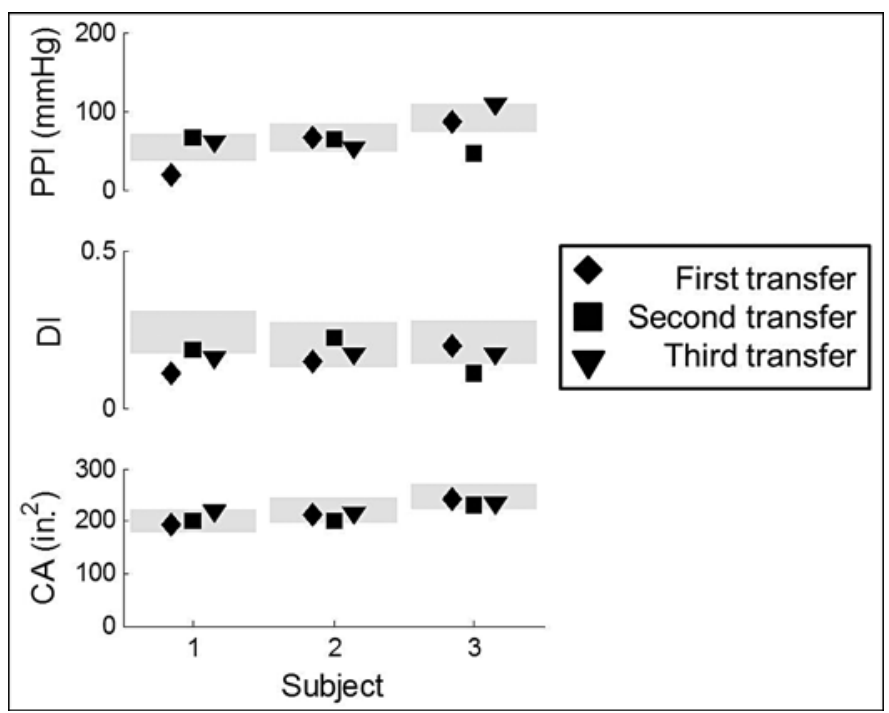

Figure 3.

After each of three transfers from wheelchair to mat platform and back, pressure distribution measurements were calculated based on interface pressure mapping. Gray bands correspond to pressure distribution measurements that could be considered equivalent to expert baseline. $\mathrm{CA}=$ contact area, $\mathrm{DI}=$ dispersion index, $\mathrm{PPI}=$ peak pressure index.

chair. Figure 5 shows the pressure distribution measurements based on IPM before and after anterior, right, and left pressure relief maneuvers. All measured relief maneuvers resulted in decreases in PPI, DI, and CA.

\section{Fittings}

Wheelchair seat fittings by the therapist subjects generally resulted in pressure distributions that were close to the expert baseline. Figure 6 shows the pressure distribution measurements based on IPM after initial and final fittings for the three subjects with SCI, each performed by three therapists. Most final fittings had better or equivalent pressure distributions than initial fittings (corresponding to lower or equivalent PPIs, lower or equivalent DIs, and higher or equivalent CAs). Thus, the full fitting process (initial and final) generally resulted in a better fit than the initial process alone. However, in many cases the initial fitting process by itself was able to create a clinically acceptable fit that was close to the expert baseline and required much less time than the full fitting process. Therapist subjects had difficulty fitting the wheelchair seat for subject 3, who had one pressure distribution with a PPI of $130 \mathrm{~mm} \mathrm{Hg}$ (40 mm Hg above the expert baseline), a DI 


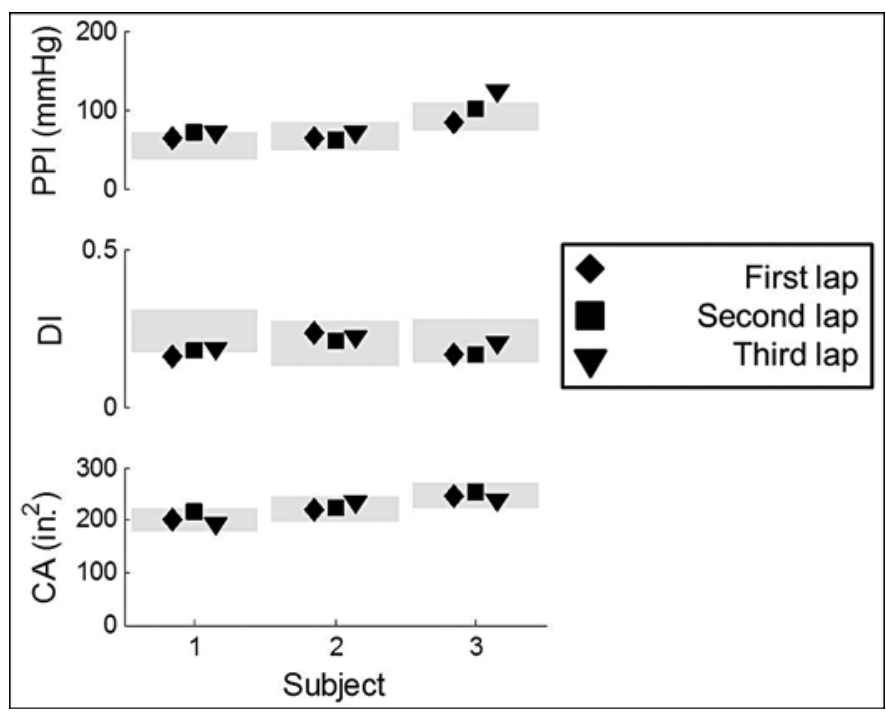

Figure 4.

After three laps of wheeling around hospital hallways, pressure distribution measurements were calculated based on interface pressure mapping. Gray bands correspond to pressure distribution measurements that could be considered equivalent to expert baseline. $\mathrm{CA}=$ contact area, $\mathrm{DI}=$ dispersion index, $\mathrm{PPI}=$ interface pressure mapping.

of 35 percent ( $14 \%$ above the expert baseline), and CA of 228 in. $^{2}$ (20 in. ${ }^{2}$ below the expert baseline). However, the therapist subjects still determined the wheelchair seat was clinically acceptable.

The time required to fit the wheelchair seat varied across subjects. Initial fittings took approximately $2 \mathrm{~min}$ for all subjects, and final fittings had mean times of 16, 7, and 25 min for subjects 1,2 , and 3, respectively. The shortest total time required to completely fit the wheelchair seat in this study was 4 min for subject 2, and the longest total time was $40 \mathrm{~min}$ for subject 3 . Some therapists were able to complete two full fittings within their hour session for subjects 1 and 2. However, no therapist was able to complete more than one fitting in the allotted time for subject 3 .

\section{DISCUSSION}

In this study, five therapist subjects were able to customize a strap-based seating system to obtain clinically acceptable pressure distributions for three Veteran subjects with SCI. Pressure distribution measurements were

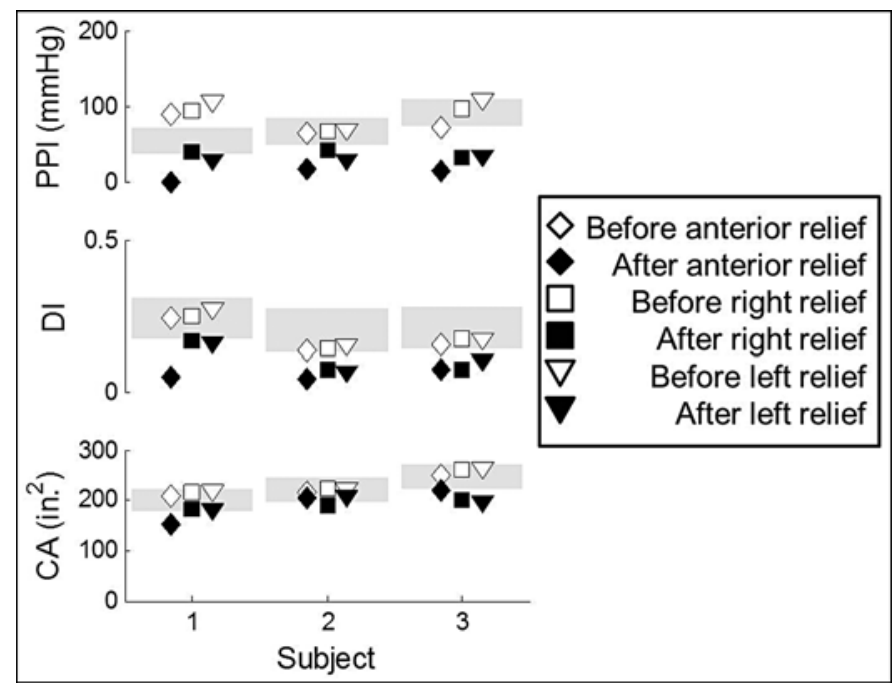

Figure 5.

Before and after three types of pressure relief maneuvers (anterior, right, and left), pressure distribution measurements were calculated based on interface pressure mapping. Gray bands correspond to pressure distribution measurements that could be considered equivalent to expert baseline. $\mathrm{CA}=$ contact area, $\mathrm{DI}=$ dispersion index, $\mathrm{PPI}=$ peak pressure index .

found to remain stable after two common wheelchair activities, wheeling and transfers. Also, the subjects were able to perform pressure relief maneuvers with the strapbased wheelchair.

All fittings resulted in seating interfaces that were clinically acceptable, which is the most important factor in evaluating wheelchair seats. Measurements of PPI, DI, and CA were used to quantify how close pressure distributions were able to get to the "expert baseline." The expert baseline was the pressure distribution for each subject with SCI on the strap-based wheelchair seat as adjusted by a highly experienced VA therapist (author B.W.) and an orthotist and coinventor of the adjustable strap-based wheelchair system (author M.P.). Measurements that were within the reliability coefficient $(17.7 \mathrm{~mm} \mathrm{Hg}, 7 \%$, and 23.7 in. $^{2}$ for PPI, DI, and CA, respectively) values from the expert baseline [7] were considered to be equivalent to the expert baseline. In most cases, the pressure distribution measurements based on IPM after fittings by the therapist subjects were equivalent or very close to the expert baseline. Similarly, measurements based on IPM after transfers and laps were equivalent to the expert baseline. It is unknown if these pressure distributions are ideal for the subjects with SCI. Nevertheless, all final fittings resulted 


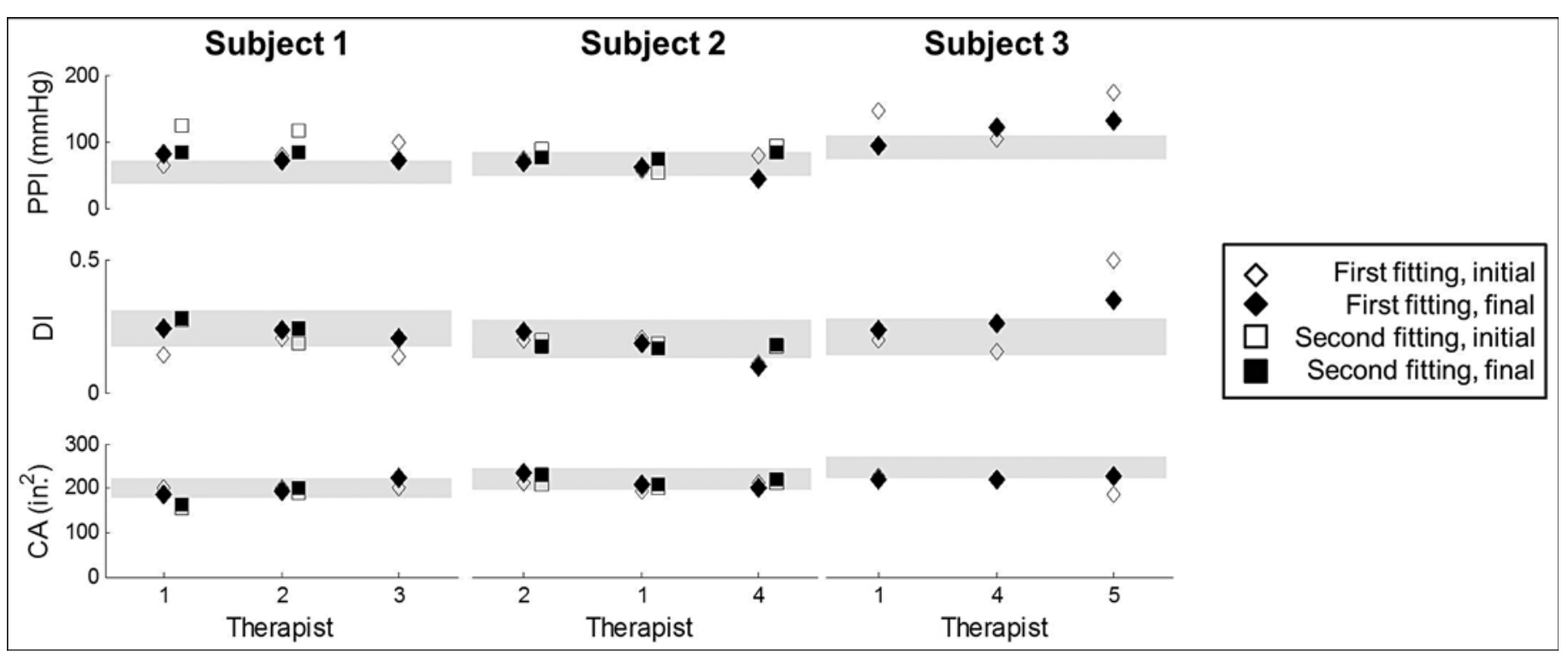

Figure 6.

After initial and final fittings, pressure distribution measurements were calculated based on interface pressure mapping. Therapists were able to perform either one or two full fittings per subject. Gray bands correspond to pressure distribution measurements that could be considered equivalent to expert baseline. $\mathrm{CA}=$ contact area, $\mathrm{DI}=$ dispersion index, $\mathrm{PPI}=$ peak pressure index.

in clinically acceptable pressure distributions and all DI measurements were well below 55 percent, a level shown to be a risk factor for ulceration [15]. Also, because IPM was not performed as the subjects with SCI sat on their regular wheelchair cushions, it is not known if the therapists would have chosen the strap-based wheelchair seat over the regular wheelchair cushion. A future study that compares strap-based wheelchair seats with standard-ofcare cushions would be necessary to determine which types of wheelchair seats are better for different patients.

One of the biggest advantages of this strap-based wheelchair seating system over other custom seating systems is the time required for therapists to customize the seat for wheelchair users. In this study, the total fitting time (including both initial and final fitting processes) had a range from 4 to $40 \mathrm{~min}$, a large time savings compared with other custom seats, which can require several weeks to be fabricated and delivered. Only "foam in place” molding systems have comparable times, but they cannot be readjusted and have concerns over toxicity. Having a short total fitting time means that wheelchair seat customization may be incorporated in a single clinical appointment. In many cases, initial fittings were able to get pressure distributions to levels comparable to the expert baseline and required only 2 min. In most other cases, a few key strap adjustments were necessary to bring the seat to an acceptable level. However, because small manual adjustments could be made continuously, many therapists spent time "tweaking" the system.

The biggest challenge in this study was using the IPM sensor mat. The sensor mat was frequently unable to conform to the contours of the strap-based wheelchair seat, resulting in folds in the mat. A large amount of time was spent by each therapist trying to flatten and adjust the pressure mat. The sensor mat folds could also have affected the accuracy of PPI, DI, and CA measurements. The difficulty in positioning the sensor mat was increased with subject 3 , whose heavier weight resulted in deeper contours. All therapists had more difficulty fitting his wheelchair seat than the other two subjects. It is uncertain whether his relatively higher PPI measurements were real and due to the wheelchair seat or if they were due to the sensor mat not fully conforming to the deeper seat contours.

Feedback from clinicians and subjects was generally positive. Clinicians liked that the system was fast to customize. They also liked being able to reconfigure the seating surface over time to adapt to changes in weight, anatomy, or new wounds as needed. All the subjects with SCI found the seat firmer than their current wheelchair seat. Several subjects felt they had better posture, but one 
subject felt the wheelchair was more difficult to transfer out of than his current wheelchair. Several subjects asked about the long-term stability of a strap-based wheelchair seat, which could be the basis of a future study. Other future studies could compare strap-based wheelchair seats with other custom and noncustom seats, evaluate the durability of the straps and seating surface, and also determine which patients would be most appropriate candidates for strap-based wheelchair seats. Additionally, future versions of the strap-based wheelchair seat could be made to be modular, such that they could be used on many different manual or power wheelchairs.

\section{CONCLUSIONS}

This study was the first clinical evaluation of the FlexForm seating system in wheelchair users with SCI. The strap-based wheelchair is a new method to provide a custom seating interface and results in a different, firmer feel than other wheelchair cushions. Common wheelchair activities, such as wheeling or transferring, did not negatively affect pressure distributions. Also, pressure relief maneuvers could be performed. For all three subjects with SCI, therapists were able to use the strap-based system to obtain clinically acceptable seating interfaces in less than an hour and in many cases just a few minutes. Future studies are necessary to look at the long-term effects of using a strap-based system, to compare it with other custom and noncustom seats, and to identify the users that could most benefit from a rapidly customizable, strap-based wheelchair seat.

\section{ACKNOWLEDGMENTS}

\author{
Author Contributions: \\ Study concept and design: J. E. Ferguson, G. D. Goldish, A. H. Hansen. \\ Acquisition of data: J. E. Ferguson, B. L. Wittig, M. Payette, \\ A. H. Hansen. \\ Analysis and interpretation of data: J. E. Ferguson, A. H. Hansen. \\ Drafting of manuscript: J. E. Ferguson, A. H. Hansen. \\ Critical revision of manuscript for important intellectual content: \\ J. E. Ferguson, B. L. Wittig, M. Payette, G. D. Goldish, A. H. Hansen. \\ Financial Disclosures: Mr. Payette is a coinventor of the FlexForm \\ seating system and an employee of Tamarack Habilitation Technolo- \\ gies Inc. \\ Funding/Support: This material was based on work supported by the \\ Minnesota Veterans Medical Research \& Education Foundation and \\ the VA, Veterans Health Administration, Office of Research and \\ Development.
}

Additional Contributions: The authors thank Dr. Stephen Sprigle at the Georgia Institute of Technology for his advice in performing interface pressure measurements. We would also like to thank Tamarack Habilitation Technologies Inc for providing wheelchairs for use in the study and for providing a wheelchair for clinical use at the Minneapolis VA Health Care System.

Institutional Review: All Veteran and therapist subjects completed the consent process approved by the Institutional Review Board of the Minneapolis VA Health Care System.

Participant Follow-Up: The authors do not plan to inform participants of the publication of this study.

Disclaimer: The contents of the publication do not necessarily represent the views of the VA or the U.S. Government.

\section{REFERENCES}

1. Richardson RR, Meyer PR Jr. Prevalence and incidence of pressure sores in acute spinal cord injuries. Paraplegia. 1981;19(4):235-47. [PMID:7290733] http://dx.doi.org/10.1038/sc.1981.47

2. Fuhrer MJ, Garber SL, Rintala DH, Clearman R, Hart KA. Pressure ulcers in community-resident persons with spinal cord injury: Prevalence and risk factors. Arch Phys Med Rehabil. 1993;74(11):1172-77. [PMID:8239957]

3. Garber SL, Rintala DH. Pressure ulcers in veterans with spinal cord injury: A retrospective study. J Rehabil Res Dev. 2003;40(5):433-41. [PMID:15080228]

http://dx.doi.org/10.1682/JRRD.2003.09.0433

4. Sprigle S, Sonenblum S. Assessing evidence supporting redistribution of pressure for pressure ulcer prevention: A review. J Rehabil Res Dev. 2011;48(3):203-13.

[PMID:21480095]

http://dx.doi.org/10.1682/JRRD.2010.05.0102

5. Ferguson-Pell MW. Seat cushion selection. J Rehabil Res Dev Clin Suppl. 1990;(2):49-73. [PMID:2370637]

6. Garber SL, Dyerly LR. Wheelchair cushions for persons with spinal cord injury: An update. Am J Occup Ther. 1991;45(6):550-54. [PMID:2063944] http://dx.doi.org/10.5014/ajot.45.6.550

7. Sprigle S, Dunlop W, Press L. Reliability of bench tests of interface pressure. Assist Technol. 2003;15(1):49-57. [PMID:14760981] http://dx.doi.org/10.1080/10400435.2003.10131889

8. Pipkin L, Sprigle S. Effect of model design, cushion construction, and interface pressure mats on interface pressure and immersion. J Rehabil Res Dev. 2008;45(6):875-82. [PMID:19009473] http://dx.doi.org/10.1682/JRRD.2007.06.0089

9. Sprigle S, Chung KC, Brubaker CE. Factors affecting seat contour characteristics. J Rehabil Res Dev. 1990;27(2): 127-34. [PMID:2366196] http://dx.doi.org/10.1682/JRRD.1990.04.0127 
10. Sprigle S, Chung KC, Brubaker CE. Reduction of sitting pressures with custom contoured cushions. J Rehabil Res Dev. 1990;27(2):135-40. [PMID:2366197] http://dx.doi.org/10.1682/JRRD.1990.04.0135

11. Tasker LH, Shapcott NG, Watkins AJ, Holland PM. The effect of seat shape on the risk of pressure ulcers using discomfort and interface pressure measurements. Prosthet Orthot Int. 2014;38(1):46-53. [PMID:23685919]

12. Tang S. Seat cushions. In: Webster JG, editor. Prevention of pressure sores: Engineering and clinical aspects. Philadelphia (PA): Adam Hilger; 1991. p. 54-74.

13. Apatsidis DP, Solomonidis SE, Michael SM. Pressure distribution at the seating interface of custom-molded wheelchair seats: Effect of various materials. Arch Phys Med Rehabil. 2002;83(8):1151-56. [PMID:12161839] http://dx.doi.org/10.1053/apmr.2002.33987

14. Lemaire ED, Upton D, Paialunga J, Martel G, Boucher J. Clinical analysis of a CAD/CAM system for custom seating: A comparison with hand-sculpting methods. J Rehabil Res Dev. 1996;33(3):311-20. [PMID:8823678]
15. Drummond D, Breed AL, Narechania R. Relationship of spine deformity and pelvic obliquity on sitting pressure distributions and decubitus ulceration. J Pediatr Orthop. 1985; 5(4):396-402. [PMID:3894415] http://dx.doi.org/10.1097/01241398-198507000-00002

Submitted for publication January 9, 2014. Accepted in revised form May 28, 2014.

This article and any supplementary material should be cited as follows:

Ferguson JE, Wittig BL, Payette M, Goldish GD, Hansen AH. Pilot study of a strap-based custom wheelchair seating system in persons with spinal cord injury. J Rehabil Res Dev. 2014;51(8):1255-64.

http://dx.doi.org/10.1682/JRRD.2014.01.0007

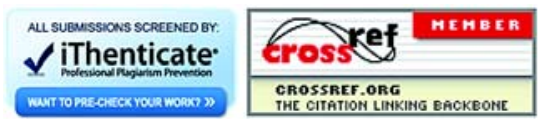


